\title{
Predictive Values of the SeLECT Score and IL-I $\beta$ for Post-Stroke Epilepsy
}

\author{
Lan Shen' \\ Jun Yang ${ }^{2}$ \\ Yueling Tang' \\ 'Department of Neurology, Central \\ Hospital of Jiangjin District, Chongqing, \\ 402260, People's Republic of China; \\ ${ }^{2}$ Department of Critical Care Medicine, \\ Central Hospital of Jiangjin District, \\ Chongqing, 402260, People's Republic of \\ China
}

Correspondence: Yueling Tang Department of Neurology, Central Hospital of Jiangin District, No. 725, Jiangzhou Road, Dingshan Street, Jiangjin District, Chongqing, 402260, People's

Republic of China

Tel +86-13667697968

Email tangy0729@I26.com
Purpose: To establish a new prognostic tool for the prediction of post-stroke epilepsy (PSE)

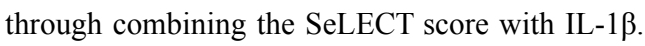

Patients and Methods: This prospective observational study included 915 patients with acute ischemic stroke. The SeLECT score was calculated, and serum IL-1 $\beta$ levels were measured within $24 \mathrm{~h}$ of their admission. One unprovoked late seizure following the acute phase of stroke was diagnosed as PSE. All patients were divided into PSE group and nonPSE group according to the occurrence of PSE. Multivariate analysis was performed to determine the independent associations between the SeLECT score, IL-1 $\beta$ and PSE. Receiver operating characteristic (ROC) curve was employed to assess the predictive values of the SeLECT score, IL-1 $\beta$ and their combination for PSE.

Results: Fifty-three patients occurred PSE within 1 year after stroke onset (5.8\%). Multivariate analysis demonstrated that the SeLECT score [odds ratio (OR): $1.416,95 \%$ confidence interval (CI): 1.191-1.863, $P=0.013$ ] and IL-1 $\beta$ (OR: 1.457, 95\% CI: 1.215 $1.894, P<0.001)$ were independent risk factors for PSE after adjusting for more than one comorbidity, stroke laterality, large-artery atherosclerosis, thrombolysis, age and use of statins. The AUC of the SeLECT score and IL-1 $\beta$ for predicting PSE was 0.756 (SE: 0.033, 95\% CI: 0.692-0.819) and 0.811 (SE: 0.032, 95\% CI: 0.748-0.875), respectively. The AUC of their combination was 0.933 (SE: 0.027, 95\% CI: 0.880-0.985). Z test showed that the AUC of their combination was significantly higher than that of the SeLECT score or IL-1 $\beta$ alone $(0.933$ vs $0.756, Z=4.151, P<0.01 ; 0.933$ vs $0.811, Z=2.914, P<0.01)$. Combination prediction of the SeLECT score and IL-1 $\beta$ for PSE had a high predictive value with a sensitivity of $88.06 \%$ and specificity of $82.37 \%$.

Conclusion: The combination of the SeLECT score and IL-1 $\beta$ had a potential to act as a new prognostic tool for the prediction of PSE.

Keywords: post-stroke epilepsy, SeLECT score, IL-1 $\beta$, predictive values

\section{Introduction}

Stroke is a common cause of seizures and epilepsy, ${ }^{1}$ accounting for $11 \%$ of all epilepsy cases and $55 \%$ of newly diagnosed seizure cases in the elderly population. ${ }^{2}$ The reported incidence rate of post-stroke epilepsy (PSE) varies considerably, ranging from $2 \%$ to $15 \%{ }^{3-8}$ This inconsistency in the reported incidence rate of PSE may be attributed to differences in PSE definitions, not distinguishing early from late seizures, not differentiating between stroke subtypes, and a large heterogeneity in the length of follow-up. PSE has been confirmed as a serious obstacle among post-stroke survivors. ${ }^{8}$ Under ischemic status, seizures can exacerbate secondary injury and influence long-term functional outcomes. ${ }^{9}$ Compared with 
seizure-free stroke survivors, patients with PSE have poor prognosis, declined quality of life and increased mortality. ${ }^{10-12}$ Therefore, it would be extremely helpful to find new prognostic tools for the prediction of PSE.

IL-1 $\beta$ is an inflammatory cytokine with constitutive expression in the central nervous system, ${ }^{13}$ and its levels significantly elevated after ischemic stroke. ${ }^{14-16}$ At the same time, elevated IL-1 $\beta$ levels have been detected in various forms of epilepsy with different etiologies. ${ }^{17}$ A recent study has demonstrated that IL- $1 \beta$ can be applied in predicting seizure recurrence after the first epileptic seizure among ischemic stroke patients. ${ }^{18}$ The SeLECT score is a novel clinical tool for the prediction of late seizures after ischemic stroke, which is developed by Galovic et al in 2018. ${ }^{19}$ This score fills a gap for an evidence-based prognostic tool that can be employed to accurately predict the risk of PSE. However, the value of the combination of the SeLECT score and IL- $1 \beta$ in predicting PSE is still not evaluated. In this study, we aimed to establish a new prognostic tool for the prediction of PSE through combining the SeLECT score with IL-1 $\beta$.

\section{Patients and Methods}

\section{Patients}

This was a prospective observational study. Between July 2018 and June 2019, consecutive patients with acute ischemic stroke were enrolled in Central Hospital of Jiangjin District, Chongqing. The inclusion criteria of participants included (1) aged 18 years or older at the time of admission; (2) acute first-ever ischemic stroke; and (3) complete medical and nursing data. The exclusion criteria included (1) transient ischemic attacks or primary haemorrhagic stroke; (2) previous history of stroke or seizures; (3) history of antiepileptic drug therapy for the prevention of seizure or other diseases (eg, psychiatric disorder and migraine); (4) potentially epileptogenic comorbidities, including intracranial tumors, history of brain surgery or severe traumatic brain injury, cerebral venous thrombosis, large cerebral aneurysms, cerebral arteriovenous malformations, hydrocephalus and cerebral vasculitis; (5) died before follow-up or lost to follow-up. This study conformed to the Declaration of Helsinki and the study protocol was permitted by the Ethical Committee of Central Hospital of Jiangjin District, Chongqing. All participants provided written informed consent.

\section{Data Collection}

Data collection was performed with the blind method. The following variables were recorded at baseline, including sex, age, drinking, smoking, hypertension, dyslipidemia, diabetes mellitus, coronary heart disease, atrial fibrillation, triacylglycerol (TG), low density lipoprotein cholesterol (LDL-C), high-density lipoprotein cholesterol (HDL-C), glycosylated hemoglobin $(\mathrm{GHb})$, and total cholesterol (TC), duration from stroke onset to admission, stroke laterality and stroke treatment (thrombolysis, antiplatelet therapy, anticoagulation therapy and use of statins). Additionally, the SeLECT score was calculated through collecting data on the National Institutes of Health Stroke Scale (NIHSS) at admission, large-artery atherosclerosis, early seizure, cortical involvement and territory of middle cerebral artery.

\section{Definitions}

Stroke was diagnosed according to World Health Organization criteria and confirmed with brain computed tomography (CT) or magnetic resonance imaging (MRI). Stroke etiology was classified according to the Trial of Org 10172 in Acute Stroke Treatment (TOAST) system, including small-vessel occlusion, cardioembolism, large-artery atherosclerosis, other determined cause and undetermined cause. ${ }^{20}$ Seizures were defined based on the ILAE classification. ${ }^{21}$ Seizure occurring within the first week after stroke onset was defined as early seizure and seizure occurring outside of the first week after stroke onset as late seizure. One unprovoked late seizure following the acute phase of stroke was sufficient for the diagnosis of PSE. All PSE diagnosis were corroborated by two experienced neurologists.

\section{Follow-Up}

All participants were followed up for seizures and epilepsy through telephone interviews and face-to-face evaluations in the outpatient department within 1 year after stroke onset. Further face-to-face evaluations were conducted to confirm the diagnosis of PSE when patients were suspected of having experienced a seizure attack or an event or symptoms that mimicked seizures. Participants who completed the follow-up were divided into PSE group and non-PSE group according to occurrence of PSE.

\section{Measurement of Serum IL-I $\beta$ Levels}

Blood samples were obtained from participants within 24 $\mathrm{h}$ of their admission and centrifuged to separate serums at 
$1000 \mathrm{~g}$ for $20 \mathrm{~min}$. The serums were then stored at $-80^{\circ} \mathrm{C}$ until measurement of serum IL-1 $\beta$ levels. A double antibody sandwich ELISA method was used to measure the IL-1 $\beta$ levels, and the Human IL- $1 \beta$ ELISA Kit was provided by jandl Biological Industrial Co., Ltd. (Shanghai, China). The lower limit of detection was $0.1 \mathrm{pg} / \mathrm{mL}$ for IL-1 $\beta$.

\section{Statistical Analysis}

Statistical analysis was performed using the SPSS version 22.0 (SPSS Inc., USA). Normality of quantitative data was evaluated with Kolmogorov-Smirnov test. Data with normal distribution were described with mean \pm standard deviation, and intergroup comparisons were performed with Student's $t$-test; and data without normal distribution were described with median (M) and interquartile range (IQR), and intergroup comparisons were performed with Mann-Whitney $U$-test. Qualitative data were described with percentages or ratios (\%), and intergroup comparisons were performed with Chi-square test. Multivariate analysis was then performed for the variables with two sided $P<0.10$ in univariate analysis. Receiver operating characteristic (ROC) curve was employed to assess the predictive values of IL-1 $\beta$, the SeLECT score and their combination. Area under curve (AUC) was compared with $\mathrm{Z}$ test. The threshold for statistical significance was set as two sided $P<0.05$.

\section{Results}

The crude cohort consisted of 1092 patients. Among them, 130 patients were excluded, including 61 for transient ischemic attacks, 18 for primary haemorrhagic stroke, 30 for previous history of stroke or seizures, 9 for intracranial tumors, 6 for history of brain surgery or severe traumatic brain injury, 4 for cerebral venous thrombosis, 2 for large cerebral aneurysms. Additionally, 47 patients were died before follow-up or lost to follow-up. Therefore, a total of 915 patients were finally included in this analysis. Overall, 53 patients occurred PSE within 1 year after stroke onset $(5.8 \%)$. All 53 PSE patients were administered with antiepileptic drugs, and 48 patients achieved the desired control effect.

\section{Univariate Analysis}

According to the results of univariate analysis (Table 1), the SeLECT score, IL-1 $\beta$, more than one comorbidity, stroke laterality, large-artery atherosclerosis and thrombolysis were statistically different between PSE group and
non-PSE group $(P<0.05)$, and the remaining variables were not statistically different $(P>0.05)$. However, age and use of statins had a $P$ value of $<0.10$.

\section{Multivariate Analysis}

The SeLECT score, IL-1 $\beta$, more than one comorbidity, stroke laterality, large-artery atherosclerosis, thrombolysis, age and use of statins were included in multivariate analysis. The results demonstrated that the SeLECT score [odds ratio (OR): 1.416, 95\% confidence interval (CI): 1.191-1.863, $P=0.013$ ] and IL-1 $\beta$ (OR: 1.457, 95\% CI: 1.215-1.894, $P<0.001)$ were independent risk factors for PSE after adjusting for more than one comorbidity, stroke laterality, large-artery atherosclerosis, thrombolysis, age and use of statins.

\section{Predictive Value}

The SeLECT score and IL-1 $\beta$ were applied in predicting PSE. According to their ROC curves (Figure 1), the AUC was 0.756 (SE: 0.033, 95\% CI: 0.692-0.819) and 0.811 (SE: 0.032, 95\% CI: 0.748-0.875), respectively. In order to improve predictive value, combination of the SeLECT score and IL- $1 \beta$ was used to predict PSE. The ROC curve was drawn with the probability derived from logistic regression model. The AUC was 0.933 (SE: 0.027, 95\% CI: 0.880-0.985) (Figure 1). Z test showed that the AUC of combination prediction of the SeLECT score and IL-1 $\beta$ for PSE was significantly higher than that of independent prediction of the SeLECT score or IL-1 $\beta$ (0.933 vs 0.756 , $\mathrm{Z}=4.151, \quad P<0.01 ; 0.933$ vs $0.811, \mathrm{Z}=2.914, \quad P<0.01)$. Combination prediction of the SeLECT score and IL-1 $\beta$ for PSE had a high predictive value with a sensitivity of $88.06 \%$ and specificity of $82.37 \%$ (Table 2 ).

\section{Discussion}

As an inevitable pathological process following ischemic stroke, inflammation is involved in brain injury caused by stroke. $^{22,23}$ In the brain, microglia are the primary inflammatory cells and modulate inflammation after ischemic stroke. ${ }^{24}$ Microglia can be activated after ischemic stroke with an obvious morphological transformation from a thin, ramified state to a large, amoeboid structure, ${ }^{25}$ which has been proved to be accompanied by release of inflammatory cytokines. ${ }^{26}$ Both experimental and clinical studies have demonstrated that neuroinflammation characterized with elevated levels of inflammatory cytokines associated with microglia activation is implicated in the pathogenesis of epilepsy. ${ }^{27-30}$ At the same time, the conditions that leads to 
Table I Univariate Analysis Between PSE Group and Non-PSE Group

\begin{tabular}{|c|c|c|c|c|c|}
\hline Variables & $\begin{array}{l}\text { All Patients } \\
(9 \mid 5)\end{array}$ & $\begin{array}{l}\text { PSE Group } \\
\text { (53) }\end{array}$ & $\begin{array}{c}\text { Non-PSE Group } \\
(\mathbf{8 6 2})\end{array}$ & $t / Z \mid \chi^{2}$ & $P$ \\
\hline Age (years, mean \pm standard) & $67.05 \pm 8.93$ & $68.92 \pm 8.19$ & $66.93 \pm 8.98$ & 1.707 & 0.090 \\
\hline Male (n, \%) & $540(59.0 \%)$ & $36(67.9 \%)$ & $504(58.5 \%)$ & 1.846 & 0.174 \\
\hline Drinking (n, \%) & $365(39.9 \%)$ & $17(32.1 \%)$ & $348(40.4 \%)$ & 1.433 & 0.231 \\
\hline Smoking (n, \%) & $228(24.9 \%)$ & $16(30.2 \%)$ & $212(24.6 \%)$ & 0.835 & 0.361 \\
\hline \multicolumn{6}{|l|}{ Comorbidity (n, \%) } \\
\hline Hypertension & $596(65.1 \%)$ & $32(60.4 \%)$ & $564(65.4 \%)$ & $0.56 \mathrm{I}$ & 0.454 \\
\hline Dyslipidemia & $184(20.1 \%)$ & $13(24.5 \%)$ & $17 \mid(\mid 9.8 \%)$ & 0.684 & 0.408 \\
\hline Diabetes mellitus & $156(17.0 \%)$ & $12(22.6 \%)$ & $144(16.7 \%)$ & 1.244 & 0.265 \\
\hline Coronary heart disease & $42(4.6 \%)$ & $3(5.7 \%)$ & $39(4.5 \%)$ & & $0.730 *$ \\
\hline Atrial fibrillation & $66(7.2 \%)$ & $6(11.3 \%)$ & $60(7.0 \%)$ & & $0.266 *$ \\
\hline More than one comorbidity & $562(6 I .4 \%)$ & $25(47.2 \%)$ & $537(62.3 \%)$ & 4.822 & 0.028 \\
\hline \multicolumn{6}{|l|}{ Laboratory examinations (mean \pm standard) } \\
\hline Total cholesterol (mmol/L) & $4.61 \pm 1.12$ & $4.82 \pm 1.14$ & $4.59 \pm 1.12$ & 1.427 & 0.160 \\
\hline Triacylglycerol (mmol/L) & $1.37 \pm 0.70$ & $1.28 \pm 0.63$ & $|.38 \pm 0.7|$ & -1.113 & 0.271 \\
\hline Low density lipoprotein cholesterol (mmol/L) & $2.86 \pm 0.95$ & $2.98 \pm 1.07$ & $2.85 \pm 0.94$ & 0.864 & 0.390 \\
\hline High density lipoprotein cholesterol (mmol/L) & $1.08 \pm 0.67$ & $0.96 \pm 0.81$ & $1.09 \pm 0.66$ & -1.145 & 0.256 \\
\hline Glycosylated hemoglobin (\%) & $5.49 \pm 2.06$ & $5.82 \pm 2.30$ & $5.47 \pm 2.03$ & 1.082 & 0.291 \\
\hline $\begin{array}{l}\text { Duration from stroke onset to admission ( } h \text {, mean } \\
\pm \text { standard) }\end{array}$ & $19.05 \pm 7.98$ & $19.57 \pm 7.52$ & $19.02 \pm 8.01$ & 0.515 & 0.619 \\
\hline \multicolumn{6}{|l|}{ Stroke laterality (n, \%) } \\
\hline Right & $446(48.7 \%)$ & $33(62.3 \%)$ & $4 \mid 3(47.9 \%)$ & \multirow[t]{2}{*}{4.117} & \multirow[t]{2}{*}{0.042} \\
\hline Left & $469(51.3 \%)$ & $20(37.7 \%)$ & $449(52.1 \%)$ & & \\
\hline \multicolumn{6}{|l|}{ TOAST classification (n, \%) } \\
\hline Large-artery atherosclerosis & $238(26.0 \%)$ & $21(39.6 \%)$ & $217(25.2 \%)$ & 5.416 & 0.020 \\
\hline Cardioembolism & $182(19.9 \%)$ & $9(\mid 7.0 \%)$ & $173(20.1 \%)$ & 0.299 & 0.585 \\
\hline Small vessel occlusion & $224(24.5 \%)$ & II (20.8\%) & $213(24.7 \%)$ & 0.423 & 0.516 \\
\hline Other determined & $55(6.0 \%)$ & $3(5.7 \%)$ & $52(6.0 \%)$ & & $1.000 *$ \\
\hline Undetermined & $216(23.6 \%)$ & $9(\mid 7.0 \%)$ & $209(24.2 \%)$ & 1.452 & 0.228 \\
\hline \multicolumn{6}{|l|}{ Stroke treatment $(\mathrm{n}, \%)$} \\
\hline Thrombolysis & $83(9.1 \%)$ & $10(18.9 \%)$ & $73(8.5 \%)$ & 6.546 & 0.011 \\
\hline Antiplatelet therapy & $640(69.9 \%)$ & $34(64.2 \%)$ & $606(70.3 \%)$ & 0.899 & 0.343 \\
\hline Anticoagulation therapy & $275(30.1 \%)$ & $20(37.7 \%)$ & $255(29.6 \%)$ & 1.579 & 0.209 \\
\hline Use of statins & $758(82.8 \%)$ & $39(73.6 \%)$ & $719(83.4 \%)$ & 3.391 & 0.066 \\
\hline
\end{tabular}

(Continued) 
Table I (Continued).

\begin{tabular}{|c|c|c|c|c|c|}
\hline Variables & $\begin{array}{c}\text { All Patients } \\
(9 \mid 5)\end{array}$ & $\begin{array}{c}\text { PSE Group } \\
\text { (53) }\end{array}$ & $\begin{array}{c}\text { Non-PSE Group } \\
\qquad(\mathbf{8 6 2})\end{array}$ & $t / Z \mid \chi^{2}$ & $P$ \\
\hline $\mathrm{IL}-\mathrm{I} \beta(\mathrm{pg} / \mathrm{mL})$ & $2.11 \pm 1.10$ & $4.16 \pm 1.92$ & $1.98 \pm 1.05$ & 8.191 & $<0.001$ \\
\hline SeLECT score (M, IQR) & $2(2)$ & $5(3)$ & 2(2) & 5.416 & $<0.001$ \\
\hline \multicolumn{6}{|c|}{ Distribution of each SeLECT subgroup (n, \%) } \\
\hline 0 & $211(23.1 \%)$ & $3(4.5 \%)$ & $208(24.1 \%)$ & & \\
\hline 1 & $196(21.4 \%)$ & $3(4.5 \%)$ & $193(22.3 \%)$ & & \\
\hline 2 & $132(14.4 \%)$ & $4(6.0 \%)$ & $128(\mid 4.8 \%)$ & & \\
\hline 3 & $102(11.1 \%)$ & $5(7.5 \%)$ & $97(11.2 \%)$ & & \\
\hline 4 & $86(9.4 \%)$ & $10(15.1 \%)$ & $76(8.8 \%)$ & & \\
\hline 5 & $95(10.4 \%)$ & $15(22.7 \%)$ & $80(9.2 \%)$ & & \\
\hline 6 & $47(5.1 \%)$ & $5(7.5 \%)$ & $42(4.8 \%)$ & & \\
\hline 7 & $27(3.0 \%)$ & $4(6.0 \%)$ & $23(2.6 \%)$ & & \\
\hline 8 & $15(1.6 \%)$ & $3(4.5 \%)$ & $12(1.3 \%)$ & & \\
\hline 9 & $4(0.4 \%)$ & $\mathrm{I}(\mathrm{I} .5 \%)$ & $3(0.3 \%)$ & & \\
\hline
\end{tabular}

Note: *Fisher's Exact Test.

Abbreviation: PSE, post-stroke epilepsy.

neuroinflammation and release of inflammatory cytokines may also facilitate epileptogenesis. ${ }^{29,30}$

IL-1 $\beta$ is mainly produced by activated microglia and astrocytes, and is a critical inflammatory cytokines during neuroinflammation after ischemic stroke. It has been detected in human cerebrospinal fluid and epileptogenic tissue, and meanwhile, it has been also demonstrated an association with the initiation and maintenance of seizures by experimental studies.

Experimental studies have demonstrated that the expression of IL-1 $\beta$ is significantly in the epileptogenic tissues of animals with epilepsy of different etiologies and in the hippocampus after seizures. ${ }^{31-35}$ Xiao et al showed that IL-1 $\beta$ was involved in the epileptogenesis of mesial temporal lobe epilepsy through inducing activation of mammalian target of rapamycin (mTOR) and subsequent activation of neurons; ${ }^{36}$ Ho et al found that peripheral inflammation caused by LPS was correlated with increased seizure susceptibility by upregulating the expression of IL$1 \beta$ in the hippocampus, ${ }^{37}$ Viviani et al demonstrated that IL-1 $\beta$ was involved in the initiation of seizures via upregulating the expression of NMDA receptors on postsynaptic cells; ${ }^{38}$ whereas Auvin et al showed that IL- $1 \beta$ receptor antagonist could inhibit the enhancement of epileptogenesis to a certain extent in immature rat brains. ${ }^{31}$ In the clinical aspect, Shi et al indicated that IL-1 $\beta$ levels in cerebrospinal fluid were higher in the epileptic pediatric population than in the controls; ${ }^{39}$ Vezzani et al demonstrated that secretion and release of IL- $1 \beta$ was remarkedly upregulated in cerebrospinal fluid and serum of epilepsy patients after tonic-clonic seizures, ${ }^{40}$ Ichiyama et al showed that IL- $1 \beta$ levels were remarkedly increased in cerebrospinal fluid of patients with febrile seizures; ${ }^{41}$ Uludag et al showed that serum IL-1 $\beta$ levels were significantly elevated in patients with temporal lobe epilepsy or extra-temporal lobe epilepsy, and there is no statistical difference in the degree of elevation of IL- $1 \beta$ between these two groups; ${ }^{42}$ Zhang et al found that upregulated expression of IL-1 $\beta$ was independently associated with increased risk of seizure recurrence after the first epileptic seizure in ischemic stroke patients, and moreover the predictive value of IL-1 $\beta$ expression levels for seizure recurrence was high, ${ }^{18}$ Choi et al found that serum IL-1 $\beta$ levels were significantly associated with disease severity in children with epilepsy, suggesting the potential of IL- $1 \beta$ as a prognostic biomarker for childhood epilepsy. ${ }^{43}$ In this 


\section{ROC Curve}

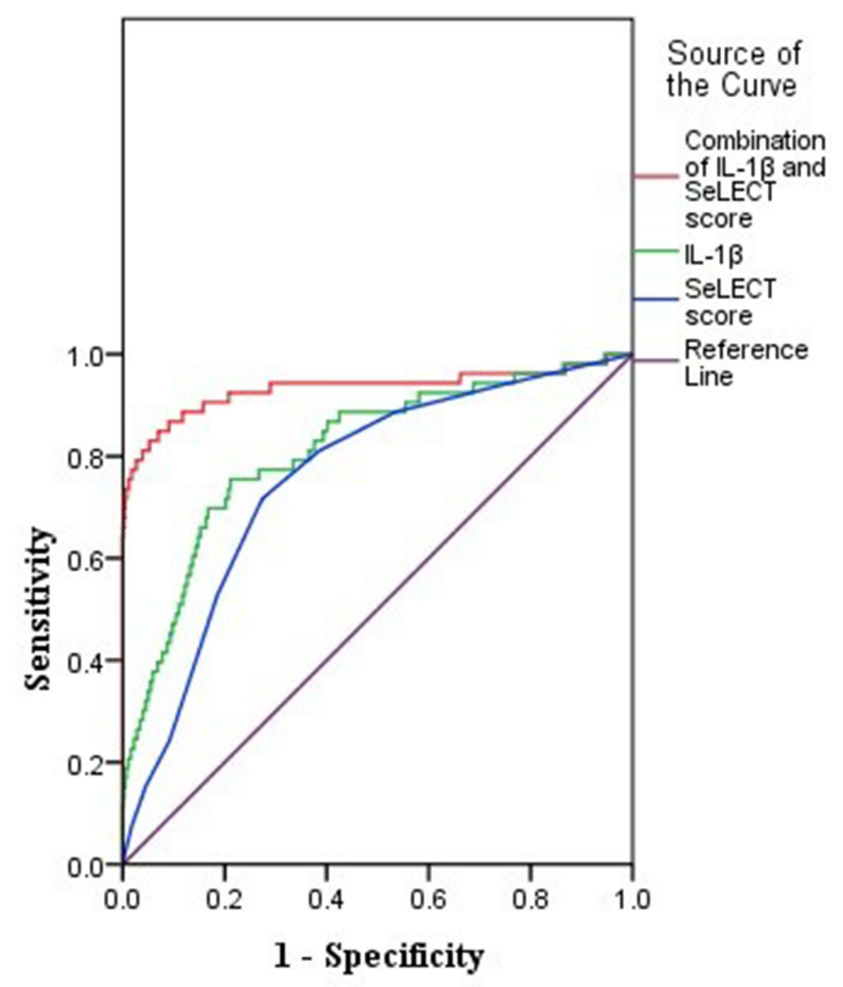

Figure I ROC curves of IL-I $\beta$, SeLECT score and their combination in predicting PSE.

study, the serum IL-1 $\beta$ level was an independent risk factor for PSE in ischemic stroke patients (OR: 1.457, 95\% CI: 1.215-1.894) and had a medium predictive value for PSE (AUC: 0.811).

The SeLECT score is a new practical prognostic tool to predict the risk of PSE, integrating five items: early seizures, severity of stroke, cortical involvement, territory of middle cerebral artery involvement and large-artery atherosclerotic aetiology. ${ }^{19}$ It has been successfully externally validated, demonstrating good discrimination and calibration. The SeLECT score ranges from 0 to 9, and higher SeLECT values indicated higher risk of PSE. The SeLECT value of 0 points is associated with a $0.7 \%$ (95\% CI $0.4-$ 1.0) risk of PSE during 1-year follow-up and a $1.3 \%(0.7-$ 1.8) risk during 5-year follow-up after ischemic stroke, whereas the value of 9 points represents a $63 \%(42-77)$ risk of PSE during 1-year follow-up and an 83\% (62-93) risk during 5-year follow-up. Therefore, the SeLECT score can be applied in identifying individuals with high risk of PSE among ischemic stroke patients. Our results further confirmed the conclusion, showing an independent association of the SeLECT score with PSE (OR: 1.416, 95\% CI: 1.191-1.863) and a medium predictive value of the SeLECT score for PSE (AUC: 0.756).

We further assessed the predictive value of the combination of the SeLECT score and IL- $1 \beta$ for PSE. The results demonstrated that the combination of the SeLECT score and IL-1 $\beta$ had a significantly improved predictive value (AUC: 0.933 ) compared with the SeLECT score or IL-1 $\beta$ alone. The sensitivity was $88.06 \%$ and the specificity was $82.37 \%$. Thus, the combination of the SeLECT score and IL-1 $\beta$ had a potential to act as a new prognostic tool for the prediction of PSE.

In this study, more than one comorbidity, stroke laterality, thrombolysis, age and use of statins were adjusted to determine the independent associations between the SeLECT score, IL-1 $\beta$ and PSE. Both the type and the dosage of statins are associated with the development of PSE, and not just the use of them. ${ }^{44}$ However, the type and the dosage of statins were not included in our analysis, this was a potential limitation. In addition, there were still two limitations in this study. The first was a small sample, especially for patients with PSE, and the other was

Table 2 Clinical Utility Indexes for the SeLECT Score, IL-I $\beta$ and Their Combination in Predicting PSE

\begin{tabular}{|l|c|c|c|c|c|c|c|c|c|}
\hline & $\begin{array}{c}\text { Best } \\
\text { Cut- } \\
\text { Off }\end{array}$ & Sensitivity & Specificity & Accuracy & $\begin{array}{c}\text { False } \\
\text { Positive } \\
\text { Rate }\end{array}$ & $\begin{array}{c}\text { False } \\
\text { Negative } \\
\text { Rate }\end{array}$ & $\begin{array}{c}\text { Positive } \\
\text { Predictice } \\
\text { Value }\end{array}$ & $\begin{array}{c}\text { Negative } \\
\text { Predictive } \\
\text { Value }\end{array}$ & $\begin{array}{c}\text { Youden } \\
\text { Index }\end{array}$ \\
\hline SeLECT score & 4 & $75.47 \%$ & $60.32 \%$ & $61.20 \%$ & $89.53 \%$ & $2.44 \%$ & $10.47 \%$ & $97.56 \%$ & 0.36 \\
\hline IL-I $\beta$ & 3.27 & $79.25 \%$ & $67.40 \%$ & $68.09 \%$ & $87.00 \%$ & $1.86 \%$ & $13.00 \%$ & $98.14 \%$ & 0.47 \\
\hline $\begin{array}{l}\text { Combination of } \\
\text { SeLECT score and } \\
\text { IL-I } \beta\end{array}$ & $88.06 \%$ & $82.37 \%$ & $82.73 \%$ & $76.38 \%$ & $0.84 \%$ & $23.62 \%$ & $99.16 \%$ & 0.70 \\
\hline
\end{tabular}


employment of the middle-term follow-up instead of a longer one.

\section{Conclusion}

Combination prediction of the SeLECT score and IL-1 $\beta$ for PSE had a high predictive value with a sensitivity of $88.06 \%$ and specificity of $82.37 \%$. Therefore, the combination of the SeLECT score and IL-1 $\beta$ had a potential to act as a new prognostic tool for the prediction of PSE.

\section{Author Contributions}

All authors made a significant contribution to the work reported, whether that is in the conception, study design, execution, acquisition of data, analysis and interpretation, or in all these areas; took part in drafting, revising or critically reviewing the article; gave final approval of the version to be published; have agreed on the journal to which the article has been submitted; and agree to be accountable for all aspects of the work.

\section{Disclosure}

All authors report no conflicts of interest for this work.

\section{References}

1. Menon B, Shorvon SD. Ischaemic stroke in adults and epilepsy. Epilepsy Res. 2009;87(1):1-11. doi:10.1016/j.eplepsyres.2009.08.007

2. Camilo O, Goldstein LB. Seizures and epilepsy after ischaemic stroke. Stroke. 2004;35(7):1769-1775. doi:10.1161/01. STR.0000130989.17100.96

3. Zelano J, Redfors P, Åsberg S, et al. Association between poststroke epilepsy and death: a nationwide cohort study. Eur Stroke J. 2016;1 (4):272-278. doi:10.1177/2396987316669000

4. Bentes C, Martins H, Peralta AR, et al. Early EEG predicts post-stroke epilepsy. Epilepsia Open. 2018;3(2):203-212. doi:10.1002/epi4.12103

5. Graham NS, Crichton S, Koutroumanidis M, et al. Incidence and associations of poststroke epilepsy: the prospective South London Stroke Register. Stroke. 2013;44(3):605-611. doi:10.1161/ STROKEAHA.111.000220

6. Guo J, Guo J, Li J, et al. Statin treatment reduces the risk of post-stroke seizures. Neurology. 2015;85(8):701-707. doi:10.1212/ WNL.0000000000001814

7. Serafini A, Gigli GL, Gregoraci G, et al. Are early seizures predictive of epilepsy after a stroke? Results of a population-based study. Neuroepidemiology. 2015;45(1):50-58. doi:10.1159/000382078

8. Zelano J. Poststroke epilepsy: update and future directions. Ther $A d v$ Neurol Disord. 2016;9(5):424-435. doi:10.1177/1756285616654423

9. Arntz RM, Maaijwee NA, Rutten-Jacobs LC, et al. Epilepsy after TIA or stroke in young patients impairs long-term functional outcome the future study. Neurology. 2013;81(22):1907-1913. doi:10.1212/01.wnl.0000436619.25532.f3

10. Arntz RM, Rutten-Jacobs LC, Maaijwee NA, et al. Poststroke epilepsy is associated with a high mortality after a stroke at young age: follow-up of transient ischemic attack and stroke patients and unelucidated risk factor evaluation study. Stroke. 2015;46(8):2309-2311. doi:10.1161/STROKEAHA.115.010115
11. Winter Y, Daneshkhah N, Galland N, et al. Health-related quality of life in patients with poststroke epilepsy. Epilepsy Behav. 2018;80:303-306. doi:10.1016/j.yebeh.2017.12.037

12. Bladin CF, Alexandrov AV, Bellavance A, et al. Seizures after stroke: a prospective multicenter study. Arch Neurol. 2000;57 (11):1617-1622. doi:10.1001/archneur.57.11.1617

13. Dinarello CA. Biologic basis for interleukin-1 in disease. Blood. 1996;87(6):2095-2147. doi:10.1182/blood.V87.6.2095.bloodjournal 8762095

14. Clausen BH, Lambertsen KL, Babcock AA, et al. Interleukin-1beta and tumor necrosis factor-alpha are expressed by different subsets of microglia and macrophages after ischemic stroke in mice. J Neuroinflammation. 2008;5(1):46. doi:10.1186/1742-2094-5-46

15. Clausen BH, Lambertsen KL, Dagnæs-Hansen F, et al. Cell therapy centered on IL-1Ra is neuroprotective in experimental stroke. Acta Neuropathol. 2016;131(5):775-791. doi:10.1007/s00401-016-1541-5

16. Davies CA, Loddick SA, Toulmond S, et al. The progression and topographic distribution of interleukin-1beta expression after permanent middle cerebral artery occlusion in the rat. J Cereb Blood Flow Metab. 1999;19(1):87-98. doi:10.1097/00004647-199901000-00010

17. de Vries EE, van den Munckhof B, Braun KP, et al. Inflammatory mediators in human epilepsy: a systematic review and meta-analysis. Neurosci Biobehav Rev. 2016;63:177-190. doi:10.1016/j. neubiorev.2016.02.007

18. Zhang Q, Li G, Zhao D, et al. Association between IL-1 $\beta$ and recurrence after the first epileptic seizure in ischemic stroke patients. Sci Rep. 2020;10(1):13505. doi:10.1038/s41598-020-70560-7

19. Galovic M, Döhler N, Erdélyi-Canavese B, et al. Prediction of late seizures after ischaemic stroke with a novel prognostic model (the SeLECT score): a multivariable prediction model development and validation study. Lancet Neurol. 2018;17(2):143-152. doi:10.1016/ S1474-4422(17)30404-0

20. Adams HP Jr, Bendixen BH, Kappelle LJ, et al. Classification of subtype of acute ischemic stroke. Definitions for use in a multicenter clinical trial. TOAST. Trial of Org 10172 in Acute Stroke Treatment. Stroke. 1993;24(1):35-41. doi:10.1161/01.str.24.1.35

21. Engel J Jr. Report of the ILAE classification core group. Epilepsia. 2006;47(9):1558-1568. doi:10.1111/j.1528-1167.2006.00215.x

22. Chamorro A, Hallenbeck J. The harms and benefits of inflammatory and immune responses in vascular disease. Stroke. 2006;37 (2):291-293. doi:10.1161/01.STR.0000200561.69611.f8

23. McColl BW, Allan SM, Rothwell NJ. Systemic infection, inflammation and acute ischemic stroke. Neuroscience. 2009;158 (3):1049-1061. doi:10.1016/j.neuroscience.2008.08.019

24. Benakis C, Garcia-Bonilla L, Iadecola C, et al. The role of microglia and myeloid immune cells in acute cerebral ischemia. Front Cell Neurosci. 2015;8:461. doi:10.3389/fncel.2014.00461

25. Patel AR, Ritzel R, McCullough LD, et al. Microglia and ischemic stroke: a double-edged sword. Int J Physiol Pathophysiol Pharmacol. 2013;5(2):73-90.

26. Ritzel RM, Patel AR, Grenier JM, et al. Functional differences between microglia and monocytes after ischemic stroke. $J$ Neuroinflammation. 2015;12(1):106. doi:10.1186/s12974-0150329-1

27. Aronica E, Crino PB. Inflammation in epilepsy: clinical observations. Epilepsia. 2011;52(Suppl 3):26-32. doi:10.1111/j.15281167.2011.03033.x

28. Janigro D, Iffland PH 2nd, Marchi N, et al. A role for inflammation in status epilepticus is revealed by a review of current therapeutic approaches. Epilepsia. 2013;Suppl 54(6):30-32. doi:10.1111/ epi. 12271

29. Godukhin OV, Levin SG, Parnyshkova EY. The effects of interleukin-10 on the development of epileptiform activity in the hippocampus induced by transient hypoxia, bicuculline, and electrical kindling. Neurosci Behav Physiol. 2009;39(7):625-631. doi:10.1007/ s11055-009-9187-6 
30. Kawamura Y, Yamazaki Y, Ohashi M, et al. Cytokine and chemokine responses in the blood and cerebrospinal fluid of patients with human herpesvirus 6B-associated acute encephalopathy with biphasic seizures and late reduced diffusion. J Med Virol. 2014;86(3):512-518. doi:10.1002/jmv.23788

31. Auvin S, Shin D, Mazarati A, et al. Inflammation induced by LPS enhances epileptogenesis in immature rat and may be partially reversed by IL1RA. Epilepsia. 2010;Suppl 51(Suppl 3):34-38. doi:10.1111/j.1528-1167.2010.02606.x

32. Strauss KI, Elisevich KV. Brain region and epilepsy-associated differences in inflammatory mediator levels in medically refractory mesial temporal lobe epilepsy. $J$ Neuroinflammation. 2016;13 (1):270. doi:10.1186/s12974-016-0727-z

33. Scorza CA, Marques MJG, Gomes da Silva S, et al. Status epilepticus does not induce acute brain inflammatory response in the Amazon rodent Proechimys, an animal model resistant to epileptogenesis. Neurosci Lett. 2018;668:169-173. doi:10.1016/j.neulet.2017.02.049

34. Han T, Qin Y, Mou C, et al. Seizure induced synaptic plasticity alteration in hippocampus is mediated by IL-1 $\beta$ receptor through PI3K/Akt pathway. Am J Transl Res. 2016;8(10):4499-4509.

35. Plata-Salamán CR, Ilyin SE, Turrin NP, et al. Kindling modulates the IL-1beta system, TNF- alpha, TGF-beta1, and neuropeptide mRNAs in specific brain regions. Brain Res Mol Brain Res. 2000;75 (2):248-258. doi:10.1016/s0169-328x(99)00306-x

36. Xiao Z, Peng J, Gan N, et al. Interleukin-1 $\beta$ plays a pivotal role via the PI3K/Akt/mTOR signaling pathway in the chronicity of mesial temporal lobe epilepsy. Neuroimmunomodulation. 2016;23(56):332-344. doi:10.1159/000460254

37. Ho YH, Lin YT, Wu CW, et al. Peripheral inflammation increases seizure susceptibility via the induction of neuroinflammation and oxidative stress in the hippocampus. J Biomed Sci. 2015;22(1):46. doi:10.1186/s12929-015-0157-8
38. Viviani B, Bartesaghi S, Gardoni F, et al. Interleukin-1beta enhances NMDA receptor-mediated intracellular calcium increase through activation of the Src family of kinases. $J$ Neurosci. 2003;23 (25):8692-8700. doi:10.1523/JNEUROSCI.23-25-08692.2003

39. Shi LM, Chen RJ, Zhang H, et al. Cerebrospinal fluid neuron specific enolase, interleukin-1 $\beta$ and erythropoietin concentrations in children after seizures. Childs Nerv Syst. 2017;33(5):805-811. doi:10.1007/ s00381-017-3359-4

40. Vezzani A, Balosso S, Ravizza T. The role of cytokines in the pathophysiology of epilepsy. Brain Behav Immun. 2008;22 (6):797-803. doi:10.1016/j.bbi.2008.03.009

41. Ichiyama T, Nishikawa M, Yoshitomi T, et al. Tumor necrosis factora, interleukin-1 $\beta$, and interleukin- 6 in cerebrospinal fluid from children with prolonged febrile seizures comparison with acute encephalitis/encephalopathy. Neurology. 1998;50(2):407-411. doi:10.1212/ wnl.50.2.407

42. Uludag IF, Duksal T, Tiftikcioglu BI, et al. IL-1 $\beta$, IL-6 and IL1Ra levels in temporal lobe epilepsy. Seizure. 2015;26:22-25. doi:10.1016/j.seizure.2015.01.009

43. Choi J, Kim SY, Kim H, et al. Serum $\alpha$-synuclein and IL-1 $\beta$ are increased and correlated with measures of disease severity in children with epilepsy: potential prognostic biomarkers? BMC Neurol. 2020;20(1):85. doi:10.1186/s12883-020-01662-y

44. Vitturi BK, Gagliardi RJ. The influence of statins on the risk of post-stroke epilepsy. Neurol Sci. 2020;41(7):1851-1857. doi:10.1007/s10072-020-04298-5
Neuropsychiatric Disease and Treatment

\section{Publish your work in this journal}

Neuropsychiatric Disease and Treatment is an international, peerreviewed journal of clinical therapeutics and pharmacology focusing on concise rapid reporting of clinical or pre-clinical studies on a range of neuropsychiatric and neurological disorders. This journal is indexed on PubMed Central, the 'PsycINFO' database and CAS, and

\section{Dovepress}

is the official journal of The International Neuropsychiatric Association (INA). The manuscript management system is completely online and includes a very quick and fair peer-review system which is all easy to use. Visit http://www.dovepress.com/testimonials.php to read real quotes from published authors. 